\title{
Change in surface states of ultrafine grained copper due to cyclic stresses
}

\author{
M. Goto ${ }^{1}$, K. Kamil ${ }^{1}$, S. Z. $\operatorname{Han}^{2}$, K. Euh ${ }^{2}$, T. Yakushiji ${ }^{3}$ \\ \& N. Kawagoishi ${ }^{4}$ \\ ${ }^{I}$ Department of Mechanical Engineering, Oita University, Japan \\ ${ }^{2}$ Korea Institute of Materials Science, Changwon, Republic of Korea \\ ${ }^{3}$ Department of Mechanical Engineering, \\ Oita National College of Technology, Japan \\ ${ }^{4}$ Department of Mechanical Engineering, Kagoshima University, Japan
}

\begin{abstract}
High-cycle fatigue tests were carried out on smooth specimens of ultrafine grained copper produced by equal channel angular pressing for 8 passes. The change in surface states during stressing was monitored, showing that shear bands (SBs) were formed at an early stage of stressing. These SBs were oriented along one set of maximum shear planes which correspond to the shear plane of the final pressing. After the formation of SBs, the surface damage was slowly extended with further stressing, where a significant extension of the damaged areas occurred in the latter half of its fatigue life. Correspondingly, the surface hardness exhibited an initially moderate drop, which was closely followed by a more severe reduction in the latter half of the fatigue life. This initial drop in hardness was found to strongly depend on the decreased dislocation density caused by the dynamic recovery due to stressing, while the more severe reduction in hardness may be attributed to the grain coarsening: coarsened grains in the post-fatigued samples were observed by the transmission electron microscopy. In addition, the growth behavior of a major crack, which led to the final fracture of the specimens, was monitored by the plastic replication technique. Fatigue cracks were initiated from SBs. The growth behavior of the major cracks was estimated quantitatively from a small-crack growth law. Keywords: copper, ultrafine grain, softening, grain coarsening, shear banding.
\end{abstract}




\section{Introduction}

The equal channel angular pressing (ECAP) technique has been preferred for producing bulk materials with ultrafine grains (UFG) in the submicron range. Over the past few decades, much work on ECAPed materials has been conducted mainly on optimizing processing condition, strengthening mechanisms, and unidirectional mechanical properties. Recently, for the envisaged structural applications, the fatigue behavior of UFG materials has been studied with a particular focus on low-cycle fatigue (LCF). UFG copper fatigued in LCF tests exhibited marked cyclic softening from the beginning of the test [1-6]. This softening appears to be attributed to the instability of its severely deformed macrostructure which leads to the formation of shear bands (SBs) and cyclic strain induced grain coarsening. Agnew and Weertman [1] studied the softening property by means of hardness measurements of post-fatigued specimens. They showed that UFG copper prepared by severe plastic deformation is susceptible to cyclic softening, where softening is found to decrease at lower strain amplitudes. In their studies, the measurements of hardness were performed on cross sections of post-fatigued specimens. Since fatigue cracks are usually initiated at the sample surface, surface damage should be studied for a better understanding of fatigue characteristics. With regard to surface damage, SBs in cyclicallydeformed UFG copper have been reported [1-4, 6-10]. Fatigue cracks are initiated in these SBs [11]. To clarify the SB formation mechanism, several investigators have studied the morphological features and microstructure of SBs by using UFG single-phase metals, with most investigators focusing on copper $(99.9-99.995 \% \mathrm{Cu})[1,2,7-12]$ as their model material. The results on the necessity of grain coarsening for SB formation are contradicting, but the purity of copper appears to significantly affect the stability of the UFG structure. Grain coarsening occurs by dynamic recrystallization, as has been suggested in previous studies $[1,2,5]$. Trace impurities impede dynamic recrystallization. For high-purity UFG copper, therefore, microstructural coarsening plays an important role in shear banding. Meanwhile, since most components of machines and structures are operated under high-cycle fatigue (HCF) regimes, the softening property of surface layers and the formation behavior of surface damage due to HCF should be studied for UFG metals that are applied to actually machine members. To date however, few studies on softening of fatigued surfaces in $\mathrm{HCF}$ regimes have been performed.

In this study, HCF tests were conducted on UFG copper. The formation behavior of surface damage and change in surface hardness during stressing were monitored. The physical background of cyclic softening is discussed later with data supplied by investigating the morphological features of surface damage and the evolution of microstructures due to stressing.

\section{Experimental procedures}

The material used was pure $(99.99 \mathrm{wt} \% \mathrm{Cu})$ oxygen-free copper. Prior to ECAP, the materials were annealed at $500^{\circ} \mathrm{C}$ for $1 \mathrm{hr}$ (grain size: $100 \mu \mathrm{m}$ ). The inner and 
outer angles of the channel intersection in the ECAP die were 90 and $45^{\circ}$, respectively. The test specimens were put through multiple ECAP cycles using the $\mathrm{Bc}$ route (after each pressing, the billet bar was rotated $90^{\circ}$ around its longitudinal axis). Eight extrusion cycles resulted in an equivalent shear strain of about 7.8 [13], and granular grains with an average size of $300 \mathrm{~nm}$ were formed (Fig. 7a). $\mathrm{MoS}_{2}$ was used as a lubricant for each pressing, and the pressing speed was $5 \mathrm{~mm} / \mathrm{sec}$. The mechanical properties before ECAP were as follows: $232 \mathrm{MPa}$ tensile strength, $65 \%$ elongation to break, and a Vickers hardness number equal to 63. After eight ECAP cycles, the properties changed to $438 \mathrm{MPa}, 28 \%$, and 141 , respectively.

Five millimeter-diameter round bar specimens (Fig. 1) were machined from the processed bars. Each specimen had a shallow circumferential notch to localize damaged areas for successive observations of the surface. However, the fatigue strength reduction factor for this geometry was close to 1 , so that each specimen can be considered as a plain specimen. All fatigue specimens were electrolytically polished $(\sim 25 \mu \mathrm{m}$ from the surface layer) in order to remove any preparation affected surface layer. All tests were carried out at room temperature using a rotating bending fatigue machine operating at $50 \mathrm{~Hz}$. Observations of fatigue damage on the specimen surface were performed using optical microscopy (OM) and scanning electron microscopy (SEM). The stress value referred to is that of the nominal stress amplitude, $\sigma_{a}$, at the minimum cross section.

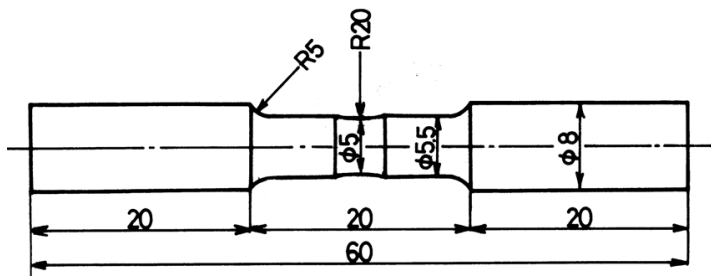

Figure 1: $\quad$ Shape of the specimen.

Transverse cross sections of the processed and post-fatigued bars were cut to prepare the specimens for transmission electron microscopy (TEM) observations. Specimens were mechanically polished to a thickness of $100 \mu \mathrm{m}$ and then subjected to twin-jet electropolishing using a solution comprised of $200 \mathrm{ml}$ of $\mathrm{CH}_{3} \mathrm{OH}$ and $100 \mathrm{ml}$ of $\mathrm{HNO}_{3}$. The jet thinning was conducted at $-30^{\circ} \mathrm{C}$.

\section{Experimental results and discussion}

Figure 2 shows the S-N diagrams of fully annealed and ECAP-treated copper. For all UFG copper examined under stress controlled testing, the enhancement in fatigue life is obvious. The degree of enhancement is sharply increased with increasing stress amplitude. In the long-life field in excess of $N=10^{7}$ cycles, however, the fatigue life to failure of UFG copper tends to coincide with that of fully annealed copper. 


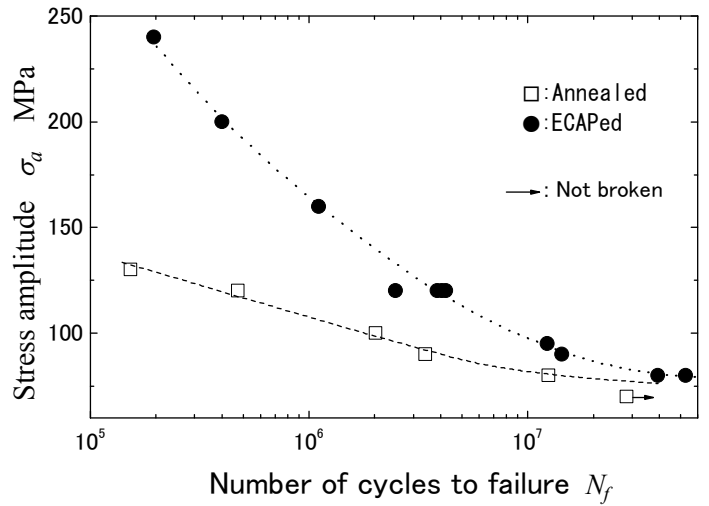

Figure 2: $\quad$ S-N curves.

Figure 3 shows the macroscopic morphological features in the surface observed at every $45^{\circ}$ along the circumferential direction. Here, the location along the circumferential direction is expressed by $\theta$ measured with an anticlockwise direction from point $\mathrm{A}$. A high population of damaged traces was observed over the surface fatigued at $\sigma_{\mathrm{a}}=240 \mathrm{MPa}$. However, their orientations depend on the value of $\theta$. Namely, the damage in the surface at $\theta=0$ and $180^{\circ}$ is oriented perpendicular to the axial (loading) direction. When $\theta=45$ and $135^{\circ}$, the orientation of damage is about $55^{\circ}$ with respect to the axial direction. However, at $\theta=90^{\circ}$ it is about $45^{\circ}$. As shown in an illustration, points $\mathrm{A}$ and $\mathrm{C}$

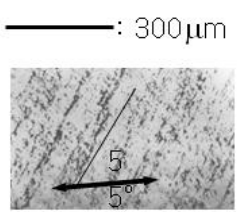

B: $\theta=45^{\circ}$

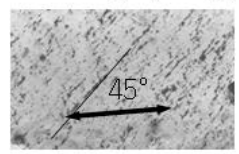

C: $\theta=90^{\circ}$

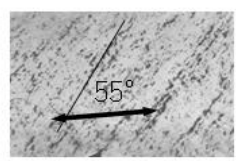

D: $\theta=135^{\circ}$

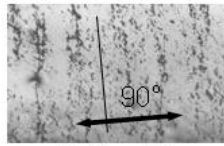

$$
\sigma_{a}=240 \mathrm{MPa}
$$

A: $\theta=0^{\circ}$

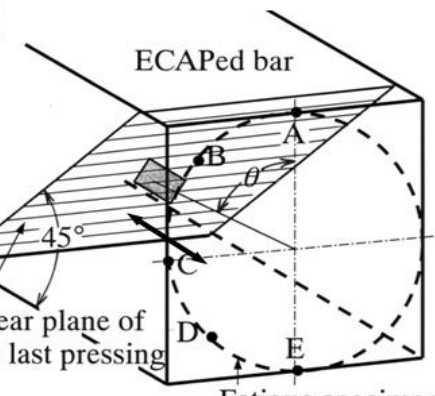

Fatigue specimen

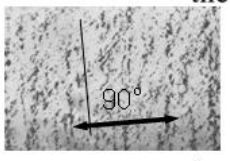

$\mathrm{E}: \theta=180^{\circ}$

\section{: Loading axis}

Figure 3: Macroscopic features of the surfaces along circumferential direction. 
of the fatigue specimen correspond to the upper and side surfaces of the ECAPed bar, and the shear plane of the last ECAP is given by the shaded lines. Thus, there is no doubt that the formation of damage is closely related to the shear plane of the last pressing. The morphology of damage is similar to the shear bands (SBs) observed by other researchers [11-13]. With regard to the macroscopic morphological features at $\sigma_{\mathrm{a}}=120 \mathrm{MPa}$, a similar tendency of the SBs orientation obtained at $\sigma_{\mathrm{a}}=240 \mathrm{MPa}$ was observed. However, the morphological features of the SBs differed from those at $\sigma_{\mathrm{a}}=240 \mathrm{MPa}$. At $\sigma_{\mathrm{a}}=$ $240 \mathrm{MPa}$, fine thin SBs were distributed with macroscopically uniform density, whereas SBs at $\sigma_{\mathrm{a}}=120 \mathrm{MPa}$ were longer and have inferior uniformity of distribution density when compared to the SBs at $\sigma_{\mathrm{a}}=240 \mathrm{MPa}$.

Figure 4 shows the change in surface Vickers hardness (load: $2.9 \mathrm{~N}$ ) during repeated stressing of $\sigma_{a}=120$ and $240 \mathrm{MPa}$. Here, each curve represents an average of the hardness measurements taken at six different locations. The maximum errors of the raw average hardness measurements were roughly within $\pm 10 \%$. The surface harnesses of post-fatigued specimens were $60-75 \%$ of asECAPed samples depending on the stress amplitudes. Regarding the decreasing trends, a surface hardness exhibited a moderate decrease in the first half of fatigue stages and a significant drop in the later stages.

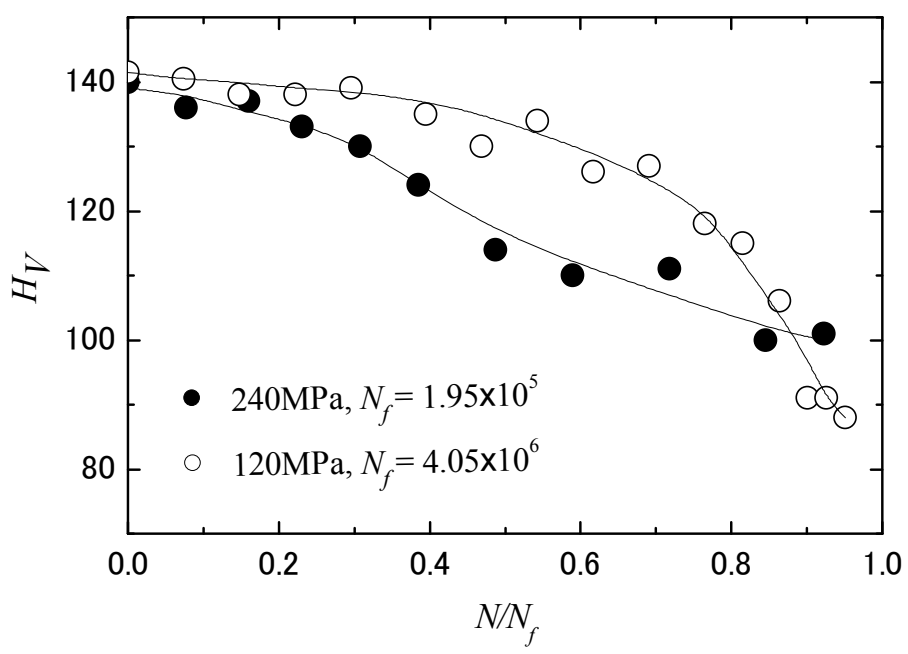

Figure 4: Change in surface hardness during stressing at $\sigma_{a}=120$ and 240 $\mathrm{MPa}$.

Figure 5 shows the formation process of surface damage for $\sigma_{a}=120$ and $240 \mathrm{MPa}$, where the number of cycles to failure, $N_{f}$, for these specimens were $N_{f}=4.05 \times 10^{6}$ and $1.95 \times 10^{5}$, respectively. During the initial stages, SBs along the plane of maximum shear stress, which correspond to the shear direction of the final pressing, were initiated. It was found that there is a pronounced time lag in 
the onset of a significant enlargement of the damaged areas [8]. For example, at $\sigma_{a}=240 \mathrm{MPa}$ (Fig.5b), the number of SBs initiated at an extremely early stage of cycling (e.g., $N / N_{f}<0.1$ ) increased as the number of cycles increased, followed by the saturation of the number of SBs after $N / N_{f} \doteqdot 0.2$. The length of SBs observed in the early fatigue stages $\left(N / N_{f}=0.2\right)$ did not increase after the subsequent stress cycling to the fracture of the specimen. Damaged areas with other morphological features started to initiate damage after $N / N_{f}=0.3$, this damage continued to grow up to the fracture, bringing a significant enlargement of the damaged areas. In addition to this, the whole surface observations of these specimens indicated that the damaged regions were formed at an early stage of cycling, and that the number and area of these regions slowly increased with further cycling up to a specific number of cycles, depending on the stress amplitude. Once this specific number of cycles had been exceeded, both the number and area of the damaged regions showed a significant rise. The number of cycles performed for $\sigma_{a}=120$ and $240 \mathrm{MPa}$ prior to the start of this remarkably large extension in damaged regions was $N / N_{f} \approx 0.5$ and 0.3 , respectively. By investigating the change in surface hardness and formation process of surface damage together, it was found that this considerably large drop in hardness in the latter half of the fatigue life is closely related to the significant formation of damaged areas.
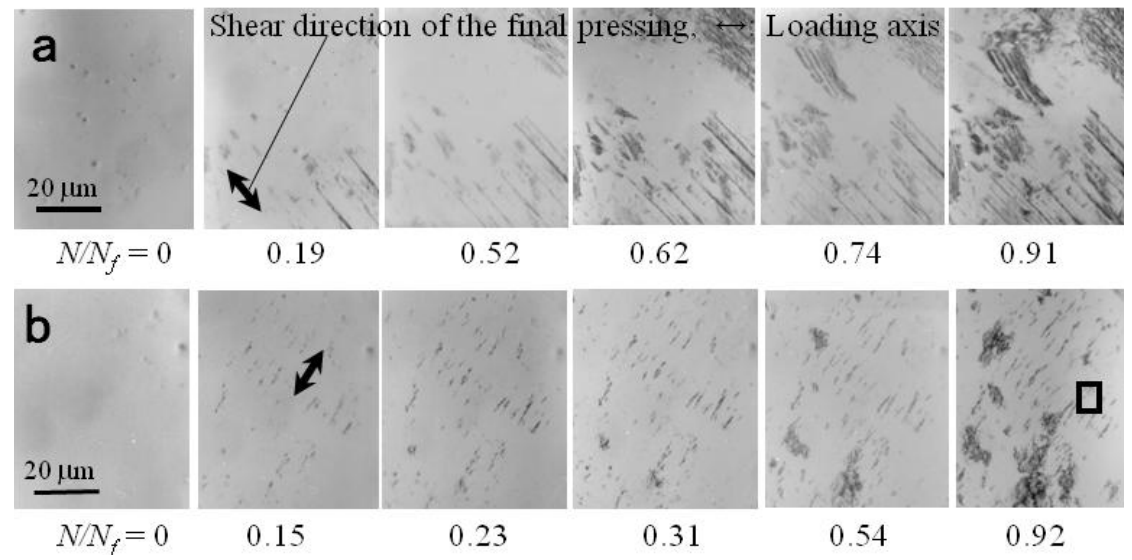

Figure 5: Change in surface states during stressing: a $\sigma_{a}=120, N_{f}=$ $4.05 \times 10^{6} ; \mathrm{b} \sigma_{a}=240 \mathrm{MPa}, N_{f}=1.95 \times 10^{5}$.

Figure 6a shows SEM micrographs of the highlighted areas (squares) indicated in Fig. 5b. The protrusions in Fig. 6a are straight and approximately parallel to the shear plane of the final pressing (as indicated by the arrow). The morphology of the protrusions would place them into the PSB-like SB category [7]. The SEM micrograph in Fig. 6b shows the formation process of PSB-like SBs during repeated stressing. Stressing for $N=7000$ cycles $\left(N / N_{f}=0.036\right)$ results in the formation of a $1 \mu \mathrm{m}$-long intrusion along the shear direction of the 
final pressing. The material could be forced to come out in the form of plate-like protrusions by way of intrusion. The formation processes of PSB-like SBs may be associated with a grain coarsening assisted by non-equilibrium states of microstructure with high energy, excess volume, and long-range stress fields. At $\sigma_{a}=120 \mathrm{MPa}$, the PSB-like SBs observed at $\sigma_{a}=240 \mathrm{MPa}$ did not form, but line-like protrusions with 20 to $40 \mu \mathrm{m}$ length were initiated [8]. A typical crack initiation site was PSB-like SBs and intrusions adjacent to the line-like protrusions.

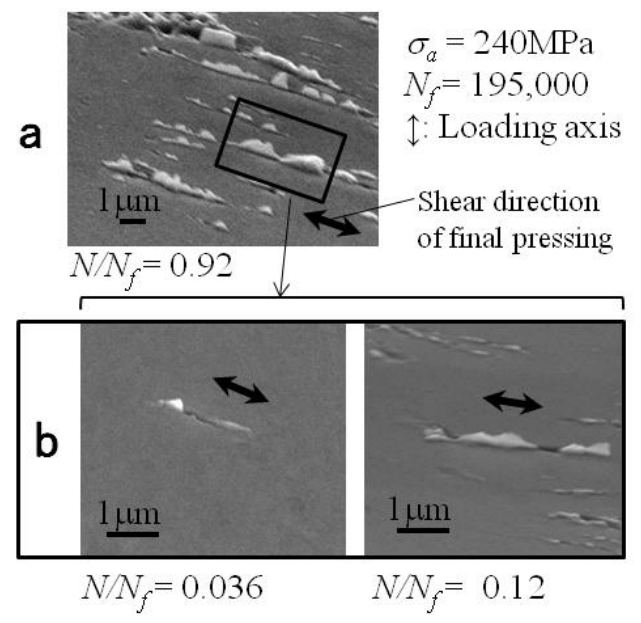

Figure 6: SEM micrographs of a fatigued surface at $\sigma_{a}=240$; a PSB-like SBs along the final pressing shear direction; $b$ formation process of PSB-like SBs.

Figure 7 shows TEM micrographs of as-ECAPed (a) and post-fatigued samples (b, c, and d). The original ECAPed microstructure had granular grains with an average size of $300 \mathrm{~nm}$. The grain coarsening occurred in the postfatigued samples. Grain coarsening occurs by dynamic recrystallization $[1,2,5]$. Evidently, the fatigue time might be important in determining the coarsening of the microstructure. It has been suggested that a certain time and cumulative strain is required for the release of sufficient stored strain energy for the dislocation motion, recrystallization process, and subsequent grain coarsening to occur $[5,12,14,15]$.

There were different decreasing trends of the $H_{v}$ vs. $N / N_{f}$ relationship between the first and later fatigue stages (Fig. 4). The hardness in the first half of the fatigue stages showed a moderate reduction. It has been shown that the GBs in UFG copper are in highly non-equilibrium conditions and have high energies, where the absorption of dislocation into GBs takes place with remarkably enhanced diffusion [16]. The initial (gentle) drop in hardness appears to result mainly from a release of strain energy relating to a decrease in the dislocation density due to dynamic recovery. Regarding the dislocation density after fatigue, 


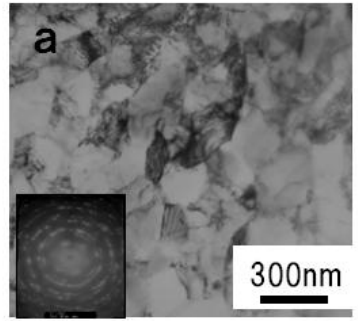

Pre-fatigue

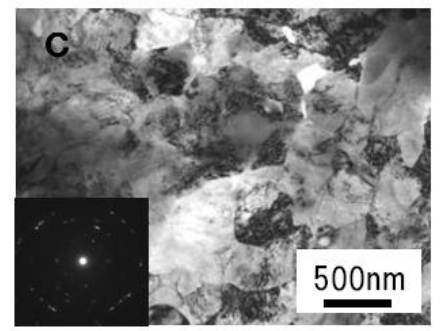

$\sigma_{a}=120 \mathrm{MPa}, N_{f}=3.87 \times 10^{6}$

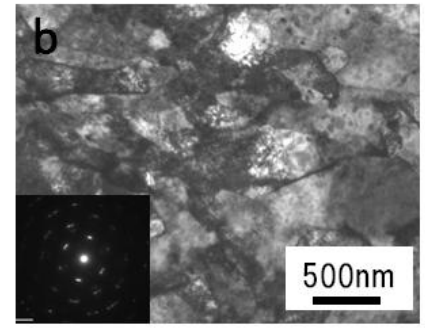

$\sigma_{a}=200 \mathrm{MPa}, N_{f}=4.01 \times 10^{5}$

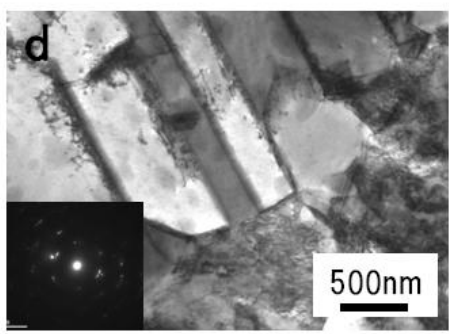

$\sigma_{a}=80 \mathrm{MPa}, N_{f}=3.94 \times 10^{7}$

Figure 7: $\quad$ TEM micrographs; (a) as-ECAPed sample; (b, c, d) coarse grains observed in post-fatigued specimens.

$\mathrm{Xu}$ et al. [17] conducted strain-controlled fatigue tests of commercial copper $(99.8 \% \mathrm{Cu})$. From TEM observations and electron backscatter diffraction grain maps, they indicated that post-fatigued structures have narrower GBs and lower dislocation density in grain interiors when compared to those in virgin microstructures. For stress-controlled fatigue tests under low plastic strain amplitude (less than $5 \times 10^{-4}$ ), Kunz et al. [9] indicated the formation of narrower GBs and lower dislocation densities in the grain interior for post-fatigued specimens of UFG copper $(99.9 \% \mathrm{Cu})$.

In the latter half of the fatigue life, all samples exhibited damaged regions that rapidly extended, with a large, simultaneous drop in surface hardness. Höppel et al. [5] conducted tension-compression fatigue tests on UFG copper to investigate the parameters governing cyclic softening and microstructural coarsening during cyclic deformation. They showed that pronounced grain coarsening is related to a marked cyclic softening in strain-controlled tests, but in the regime of low stress amplitudes in stress-controlled tests, only weak cyclic grain coarsening occurs. Mughrabi and Höppel [14] recently reviewed the fatigue properties of very finegrained materials, and noted that, while SBs have been observed in UFG copper of different purity grades after fatigue in both strain- and stress-controls, grain coarsening was essentially only observed after strain-controlled fatigue. In the present stress-controlled tests, despite the very low applied stress amplitude ( $\sigma_{a}=$ $80 \mathrm{MPa}$ : about $18 \%$ of tensile strength), coarsened grains embedded within the original fine grain/cell regions were generated after $3.94 \times 10^{7}$ repetitions (Fig. 7d). Thus, it can be concluded that the significant drop in later stages 
$\left(N>0.5 N_{f}\right.$ for $\sigma_{a}=120 \mathrm{MPa}$ and $N>0.3 N_{f}$ for $\left.\sigma_{a}=240 \mathrm{MPa}\right)$ was attributed to the grain coarsening during cyclic stressing.

Figure 8 shows the growth behaviour of an initial crack at $\sigma_{a}=120 \mathrm{MPa}$. After initiation, the crack tends to propagate along the shear direction of the final pressing.

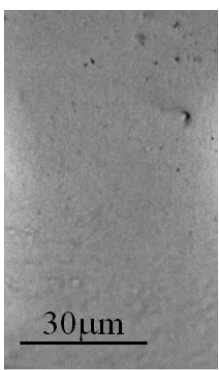

$N=0$

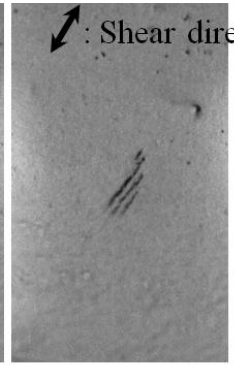

$3 \times 10^{5}$

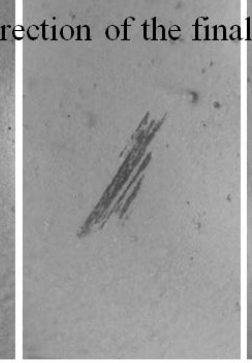

$4.5 \times 10^{5}$

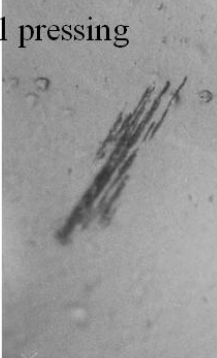

$6 \times 10^{5}$

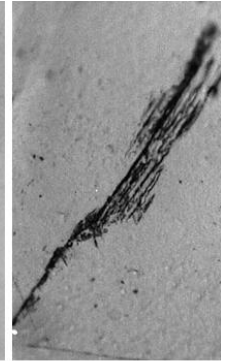

$9 \times 10^{5}$

Figure 8: The growth behavior of an initial crack at $\sigma_{a}=120 \mathrm{MPa}\left(N_{f}=\right.$ $\left.2.50 \times 10^{6}\right)$.

Figure 9 shows the crack growth curve $(\ln l$ vs. $N)$. The crack growth life form an initial size (e.g. $20 \mu \mathrm{m}$ ) to fracture accounted for about $60-80 \%$ of the fatigue life. The growth curves were approximated by linear relations, meaning that the crack growth rate is proportional to the crack length.

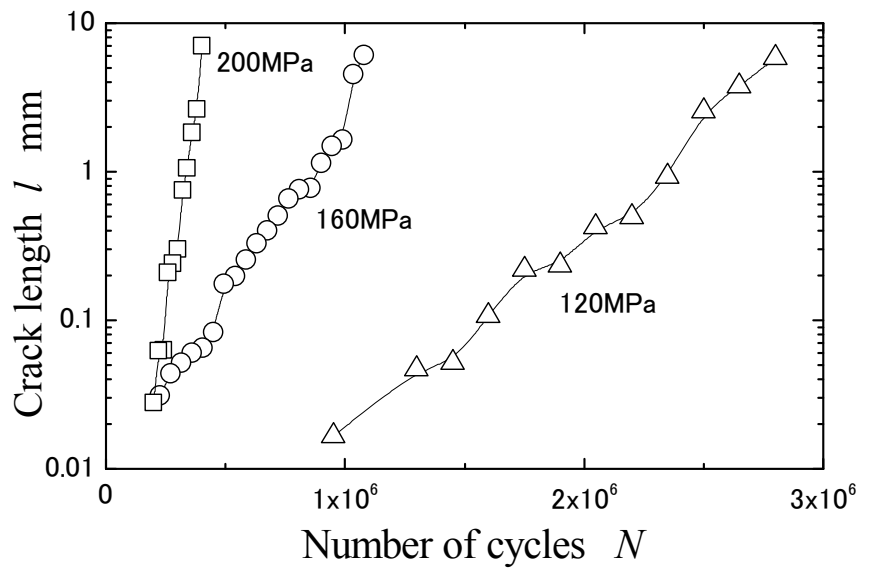

Figure 9: The crack growth curves.

It is generally accepted that the crack growth rate of a small crack in conventional grain sized materials cannot be determined by the linear elastic fracture mechanics approach. In such cases, a term $\sigma_{a}{ }^{n} l$ can evaluate the growth rate of small cracks. Here, $n$ is the material constant. Figure 10 shows the growth 
data of a small crack; $d l / d N$ vs. $\sigma_{a}{ }^{n} l$ relation $[18,19]$. The value of $n$ was 4.4 . Here, the term $d l / d N$ indicates the growth rate of major cracks calculated from the growth curves represented by smooth curves which pass through the mean average of each set of plots, rather than from the raw growth data. The growth rate of small cracks of UFG copper was estimated by $\sigma_{a}{ }^{n} l$.

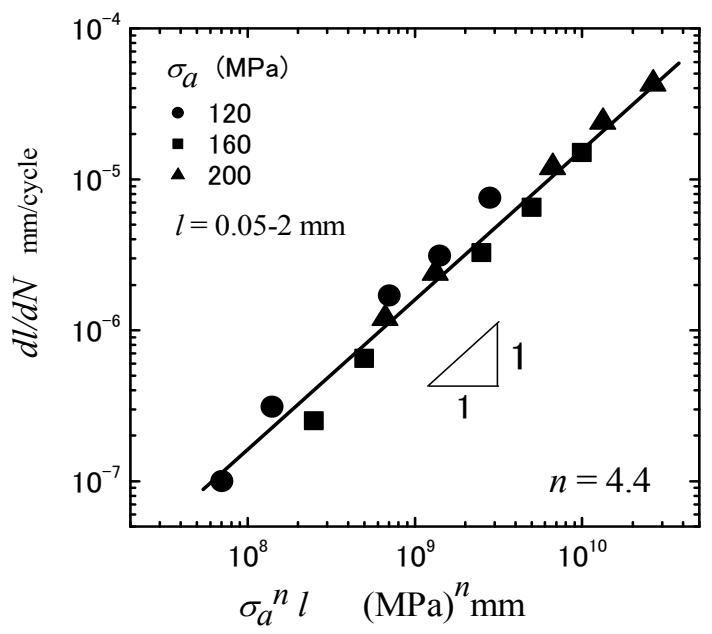

Figure 10: $\quad$ Crack growth data ( $d l d N$ vs. $\sigma_{a}{ }^{n} l$ relation).

\section{Conclusions}

The main results of the present study are summarized as follows:

(1) SBs along the shear plane of the last pressing correspond to the plane of maximum shear stress were formed at an early stage of cycling.

(2) The surface damage of UFG copper gradually formed with cycling, where a significant extension of the damaged areas occurred in the latter half of its fatigue life. Correspondingly, the surface hardness exhibited an initially moderate drop, which was closely followed by a more severe reduction in the latter half of the fatigue life.

(3) The reason for the moderate hardness drop appears to result mainly from a decrease in dislocation density due to dynamic recovery. The significant drop in the later fatigue stages was associated with grain coarsening assisted by non-equilibrium states of microstructure with high energy, excess volume, and long-range stress fields.

(4) Fatigue damage in the latter stages might be formed within the coarsened microstructure.

(5) The growth rate of small cracks could be approximately estimated by a term, $\sigma_{a}{ }^{n} l$ (with $n=4.4$ ). 


\section{Acknowledgements}

This study was supported by a Grant-in-Aid (23560093) for Scientific Research (C) from the Ministry of Education, Science and Culture of Japan as well as a grant from the Fundamental R\&D Program for Core Technology of Materials funded by the Ministry of Commerce, Industry and Energy, Republic of Korea.

\section{References}

[1] Agnew, S.R., Weertman, J.R., Cyclic softening of ultrafine grain copper. Materials Science Engineering, A244, pp. 145-153, 1998.

[2] Agnew, S.J., Vinogradov, A., Hashimoto, S., Weetman, J.R., Overview of fatigue performance of $\mathrm{Cu}$ processed by severe plastic deformation. $J$. Electonic Materials, 28, pp. 1038-1044, 1999.

[3] Vinogradov, A., Hashimoto, S., Multiscale phenomena in fatigue of ultrafine grain materials-an overview. Materials Transactions, 42, pp. 42:74-84, 2000.

[4] Hashimoto, S., Kaneko, Y., Kitagawa, K., Vinogradov, A., Valiev, R.Z., On the cyclic behavior of ultra-fine grained copper produced by equichannel angular pressing. Materials Science Forum, 312-314, pp. 593-598, 1999.

[5] Höppel, H.W., Zhou, Z.M., Mughrabi, H., Valiev, R.Z., Microstructural study of the parameters governing coarsening and cyclic softening in fatigued ultrafine-grained copper, Philosophica. Magazine, A 82, pp.17811794, 2002.

[6] Maier, H.J., Gabor, P., Karaman, I., Cyclic stress-strain response and lowcycle fatigue damage in ultrafine grained copper, Materials Science Engineering, A410-11, pp. 457-461, 2005.

[7] Wu, S.D., Wang, Z.G., Jiang, C.B., Li, G.Y., Alexandrov, I.V., Valiev, R.Z., The formation of PSB-like shear bands in cyclically deformed ultrafine grained copper processed by ECAP, Scripta Materialia, 48, pp. 1605-1609, 2003.

[8] Goto, M., Han, S,Z, Kim, S,S, Kawagoishi, N., Lim, C.Y., Significance of non-equilibrium grain boundaries in surface damage formation of ultrafinegrained copper in high-cycle fatigue, Scripta Materialia, 57, pp. 293-296, 2007.

[9] Kunz, L., Lukáš, P., Svoboda, M., Fatigue strength, microstructural stability and strain localization in ultrafine-grained copper, Materials Science Engineering, A424, pp. 97-104, 2006.

[10] Furukawa, Y., Fujii, T., Onaka, S., Kato, M., Cyclic deformation behaviour of ultra-fine grained copper processed by equal channel angular pressing, Materials. Transactions, 50, pp. 70-75, 2009.

[11] Goto, M., Han, S.Z., Euh, K., Kang, J-H., Kim, S.S., Kawagoishi, N., Formation of a high-cycle fatigue fracture surface and a crack growth 
mechanism of ultrafine-grained copper with different stages of microstructural evolution, Acta Materia 58, pp.6294-6305, 2010.

[12] Zhang, K., Weertman, J.R., Microstructural Changes produced by fatigue in high-purity copper severely deformed by cryogenic rolling, Metallurgical \&. Materials Transactions, 40A, pp. 2255-2263, 2009.

[13] Iwahashi, Y., Wang, J., Horita, Z., Nemoto, M., Langdon, T.G., Principle of equal-channel angular pressing for the processing of ultra-fine grained materials. Scripta Materialia, 35, pp. 143-146, 1995.

[14] Mughrabi, H., Höppel, H.W., Cyclic deformation and fatigue properties of very fine-grained metals and alloys, International Journal of Fatigue, 32, pp. 1413-27, 2010.

[15] Khatibi, G., Horky, J., Weiss, B., Zehetbauer, M.J., High cycle fatigue behavior of copper deformed by high pressure torsion, International Journal of Fatigue, 32, pp. 269-78, 2010.

[16] Valiev, R.Z., Kozlov, E.V., Ivanov, Yu. F., Lian, J., Nazarov, A.A., Baudelet, B., Deformation behaviour of ultra-fine grained copper. Acta Metall. Mater., 42, pp. 2467-2475, 1994.

[17] Xu, C., Wang, Q., Zheng, M., Li, J., Huang, M., Jia, Q., Zhu, J., Kunz, L., Buksa, M., Fatigue behavior and damage characteristic of ultra-fine grain low-purity copper processed by equal-channel angular pressing (ECAP), Materials Science Engineering, A475, pp. 249-256, 2008.

[18] Nisitani, H., Goto, M., Kawagoishi, N., A small-crack growth law and its related phenomena, Engineering Fracture Mechanics, 41, pp. 499-513, 1992.

[19] Goto, M., Nisitani, H., Fatigue life prediction of heat-treated carbon steels and low alloy steels based on a small crack growth law, Fatigue Fracture Engineering Materials Structures, 17, pp. 171-185, 1994. 